\title{
Participatory Design with Individuals who have Amnesia
}

\author{
Mike Wu \\ University of Toronto \\ 40 St. George Street, BA5167 \\ Toronto, Ontario, M5S 2E4 \\ 416-946-8874 \\ mchi@dgp.toronto.edu
}

\author{
Brian Richards \\ Baycrest Centre for Geriatric Care \\ 3560 Bathurst Street \\ Toronto, Ontario, M6A 2E1 \\ 416-785-2500 x2764 \\ brichards@baycrest.org
}

\author{
Ron Baecker \\ University of Toronto \\ 40 St. George Street, BA7228 \\ Toronto, Ontario, M5S 2E4 \\ 416-978-6983 \\ rmb@kmdi.toronto.edu
}

\begin{abstract}
We present experiences and insights into participatory design with individuals who have anterograde amnesia and therefore have extreme difficulty storing new memories. We discuss our design of the design process, and present a set of techniques used to support memory during and between design sessions. From this experience, we identify cognitive assumptions of participatory design that break down when working with amnestics. We generalize these ideas into an analytical framework for researchers and practitioners who intend to use participatory design with persons having various kinds of cognitive impairments. We illustrate the framework by analyzing a cognitive deficit unrelated to memory that we encountered, and an unanticipated benefit from what at first appeared to be a liability in working with this design team.
\end{abstract}

\section{Categories and Subject Descriptors}

K.4.2 [Computers and Society]: Social Issues - Assistive technologies for persons with disabilities.

\section{General Terms}

Human factors, design.

\section{Keywords}

Participatory design, user centred design, anterograde amnesia, users with disabilities, assistive technologies, cognitive prosthetics, memory aids, Personal Digital Assistants.

\section{INTRODUCTION}

Anterograde amnesia [5] is a selective memory deficit that severely impairs an individual's ability to store new facts or events. This impairment is caused by brain injury and makes difficult the transfer of new experiences into long-term memory. In contrast, memories formed prior to the brain injury (such as autobiographical information or accumulated knowledge) are recalled more effectively. However, recall of information from long term memory is often incomplete, with older memories being more accessible than memories closer in time to the injury. In

Permission to make digital or hard copies of all or part of this work for personal or classroom use is granted without fee provided that copies are not made or distributed for profit or commercial advantage and that copies bear this notice and the full citation on the first page. To copy otherwise, or republish, to post on servers or to redistribute to lists, requires prior specific permission and/or a fee.

Proceedings Participatory Design Conference 2004, Toronto, Canada. Copyright 2004 ACM 1-58113-851-2/04/07 ...\$5.00. addition procedural skills (such as typing) are typically preserved. As with other cognitive disabilities, there is a range of deficiency. Some amnestics are able to retain a general impression and partial details of a new event, while others are unable to recall what was said at the beginning of a conversation.

The impact of such memory loss can be devastating as it impedes even the simplest of day-to-day activities. Simple tasks involving memory that were easily and naturally performed before injury often become an immense struggle and challenge for amnestics. For example, social gatherings require full concentration and energy, as amnestics must keep on their mental toes in an attempt to compensate for their memory deficits. Amnestic individuals have preserved intellectual ability and social skills, consequently others are not automatically alerted to the presence of a memory deficit. To them, a person suffering from amnesia may appear somewhat rude for not recognizing a face, or odd for repeating a story that was told only minutes ago. This fuels an amnestic's feelings of anxiety, depression and low self-esteem. Social isolation is all too often an unfortunate consequence.

Although we conjectured that amnestics could nonetheless be valuable participants in design, this was not at all obvious. It was also necessary to think how techniques and methodologies could be adapted to accommodate and support memory for amnestic participants of participatory design teams. In recent years, there has been occasional use of participatory design of assistive technologies with users having cognitive deficits (see below). However, most participatory design techniques have been applied to populations having normal cognitive functions, and therefore many researchers have made assumptions that are not valid for persons having special needs. We would like to analyze these assumptions and examine how they can be used to suggest aspects of the design process that need to be adjusted and customized.

In this paper, we present a case study of participatory design of a "memory aid" with people who have amnesia, focusing on the techniques and methodologies that we have employed rather than the object of our design. From this case study, we proceed to identify assumptions in the participatory design literature that may not hold when working with persons with cognitive impairments. Participatory design can still be effective if techniques are carefully adapted and designed to address specific cognitive needs. To this end, we construct a framework that outlines this space of design, with our case study being one particular illustration of what can be achieved with amnestics. We further present two examples of cognitive issues (one related to memory and the other unrelated) that we encountered in our case study and show how they relate to the framework. This suggests that the framework may be relevant to researchers and 
practitioners using participatory design with people across the spectrum of cognitive impairments.

\section{RELATED WORK}

To situate our work, we explore how participatory design has been used with populations having special needs.

Children have preferences and needs that are not necessarily the same as those of adults [9]. Druin et al. [6, 7, 8] have done significant work in developing a form of contextual inquiry for designing with children. These techniques evolved in recognition that the methodologies of participatory design, contextual design, and cooperative design for adults need to be adapted to address the needs of children in the design team. This work illustrates that the participants' special needs can considerably impact the participatory design process.

Though many researchers have developed assistive technologies for people having various cognitive disabilities [14], most systems have not been shaped using a participatory design approach. There are a few notable exceptions:

Cole et al. [2, 3] have explored interface design with traumatic brain injured patients by using a single subject case study approach commonly applied in cognitive rehabilitation [23]. Patients guided the designers in decisions about interface parameters such as text and instructions. Clinicians were involved in design sessions focused upon correcting interface characteristics. A clear majority of the accepted interface changes and functionality in the final product were requested by the patients and clinicians during the development of the system.

Fischer et al. [10] reported on what they termed a "participatory approach" to design transportation systems for persons having cognitive disabilities. Their research methodology involved conducting field studies that examine socio-technical solutions in light of real world constraints and cognitive issues. Though their design team was composed of individuals from a very diverse set of stakeholder communities (including assistive care specialists, family support organizations, urban transportation experts, technology designers, and university researchers), the group did not include any persons who had cognitive impairments.

More recently, McGrenere et al. [16, 17] have begun the design of assistive technologies while working with persons who have aphasia, a cognitive disorder that impairs speech and language. They made two observations relating aphasics to the design process. First, the fidelity of their prototypes had a very large impact on aphasic individuals. Second, by using non-aphasic participants to help solve general usability problems, the time with aphasics could be spent focusing on language-specific issues.

There are two dimensions along which these projects are situated. They are illustrated through the following questions: (1) To what degree does the cognitively impaired user influence design, and (2) how many impaired users are included in the design team?

All the above investigations have concluded that no single perspective or technique can yield a complete solution. These projects have been deemed "participatory" by having the disabled user or related stakeholders play a role in the initial design and on-going redesign of the system. Yet in many cases, design teams involving users with disabilities were kept to single-subject sessions (of researcher and user) because the variability of the disorders were extraordinarily wide-ranging, thereby making collaboration between impaired users extremely difficult to manage and operate.

Our research builds upon these important explorations, but may be distinguished from them methodologically. We attempt to more intimately involve cognitively-impaired individuals by giving them the ability to make key decisions by consensus throughout the design lifecycle rather than just influencing external designers through suggestions/feedback at various stages of design. We achieve this by creating a design team that includes six cognitively-impaired participants who actively engage in collaborative design discussions. This is very different from prior research (which typically involves only a researcher and a single subject in design sessions). By bringing together a number of memory-impaired individuals, we follow more strongly the philosophy of participatory design than have past researchers.

Furthermore, because such severe memory disorders present unique challenges to group work, it is not clear what techniques can be used with people who have amnesia. We overcame these challenges by using a combination of design techniques that were carefully adapted to accommodate these special cognitive needs.

\section{CASE STUDY}

The Baycrest Centre for Geriatric Care and the Knowledge Media Design Institute of the University of Toronto have begun a joint project which involves the design of a memory aid application for amnestics. We have gathered together a multidisciplinary team in this endeavor, and have applied participatory design methodologies as well as general user-centered techniques over a 3 month period.

\subsection{Team Composition}

Our team consisted of six amnestics, one rehabilitation specialist (second author), and one computer scientist (first author). We very much embraced the notion of mutual learning [1], where all participants contribute their experiences and knowledge, and all learn from one another. Our design team was diverse in age (ranging from 25-55) and past occupations (including a judge and power tools designer), thus each person had unique perspectives and different expertise. Of course, our amnestics provided many first-hand experiences and insights into their memory difficulties.

All the amnestics in our group have been living with amnesia for some time and are aware of their cognitive strengths and weaknesses. All were assessed by a neurologist, a neuropsychologist, and an occupational therapist prior to their involvement in this project. The team size and composition was chosen with full consideration given to group dynamics.

The six amnestic participants were selected from a larger group of amnestics who attend Baycrest for memory-retraining and participation in a psychoeducational support group. These participants are familiar with each other because of repeated exposure to the group during the psychoeducational support sessions.

We decided upon this team composition rather than a singlesubject or a larger group in order to establish a critical mass conducive to brainstorming, and a context where participants could play off other members' ideas. Having multiple team members also meant that design decisions could be made by consensus. This led us to select amnestic participants whose level 
of memory function enabled them to retain some memory for workable periods of time, rather than involving the most severely impaired amnestics who were unable to retain information for more than a few minutes.

In addition to relying upon other abilities such as good organization skills, a person with amnesia will often depend heavily on external aids, such as friends or family. A dependence on a family member or caregiver is a common theme, often resulting in stress for the care provider and embarrassment and agitation for the amnestic. We did not include family members or caregivers in our design team. We were not concerned about the openness of the amnestics around their family, but we were conscious of the sensitivity of caregivers who may be unwilling to say something in front of the amnestics for fear of offending them. In order to capture the valuable information and insights that caregivers possess, we decided to occasionally invite them as guests to our meetings to solicit their feedback to our designs. We also chose to include them in interviews and site visits that were carried out in parallel to the design sessions.

\subsection{Interviews and Site Visits}

In parallel to the activities of the participatory design team, we conducted interviews with several amnestics and their family members to gain insights for needs analysis and requirements synthesis. A total of 18 people participated in interviews: 8 amnestics, 8 relatives (of the amnestics), 1 health care worker, and 1 occupational therapist (who works closely with a large number of amnestics). Some of the amnestics interviewed were a part of our design group while others who participated were not. The interviews were conducted as part of eight site visits by a researcher (first author) to the residences of each family. There are two ways in which we used this information.

First, we intended to use the information gathered from this usercentered technique in the participatory design group for discussion. One consideration was that of the sensitivity of the data. Some of the data was gathered from our design partners who may feel embarrassed if details were revealed, even if they are not singled out. To avoid this potential problem, we decided that only the general issues and insights would be shared by the researchers without indication that it was gathered from a specific interview.

Second, some of the insights gathered from these interviews and site visits have led to modifications that explicitly and implicitly support memory during design meetings and in between meetings. Key insights gathered from the interviews and site visits are discussed in context in the next sections of the paper (see [26] for additional detail).

\subsection{Designing the Process}

The design team participated in early design stages involving: definition of problem statement and goals, requirements analysis, concept development, and high-level and low-level design. Every week, we held a participatory design session lasting 1 to 2 hours. We ran these sessions over a period of 3 months. A breakdown of the design stages over time can be seen in Table 1.

In the next few sections, we elaborate how we have architected the design process to accommodate working with amnestics. We present four techniques that we used to directly support memory during and in between design sessions. Table 2 summarizes the techniques.

Table 1. The design stages in our case study.

\begin{tabular}{|c|c|}
\hline Design Stage & Week \\
\hline Project Overview and Goals & 1 \\
\hline Needs Analysis & 2 \\
\hline Requirements Analysis & $3-4$ \\
\hline Concept Development & 5 \\
\hline High-Level Design & $6-8$ \\
\hline Low-Level Design & $9-12$ \\
\hline
\end{tabular}

Table 2. Techniques used to support memory during and between design sessions.

\begin{tabular}{|c|c|c|}
\hline Techniques Supporting Memory & 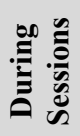 & 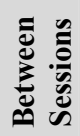 \\
\hline Incorporating Structure in Review and Activity & $\checkmark$ & $\checkmark$ \\
\hline Creating Environmental Support & $\checkmark$ & $\checkmark$ \\
\hline Emphasizing Physical Artifacts & $\checkmark$ & $\checkmark$ \\
\hline Documenting Design History & $x$ & $\checkmark$ \\
\hline
\end{tabular}

3.3.1 Incorporating Structure in Review and Activity

An amnestic's general sense of a presented fact is often available during the entirety of the design session, but the details quickly fade or degrade over time. For example, in the middle of a meeting, one of our amnestics would know that we spoke about interface components at the beginning of the session but would not be able to recall what was said about those components. It is important to address this as many design decisions are based on a collection of arguments that may be presented over a lengthy period of time.

The most obvious solution to this problem is to review and revisit items throughout a meeting, using redundancy to advantage. Going over the material and design decisions increases the chances that the content becomes familiar to the amnestics. Also, we have found through our interviews that presenting details at a later stage can help trigger recall of the larger memory encompassing those details. We made use of three reviewing techniques:

1. Review to Prepare (for supporting memory during session). At the beginning of each session, we verbally reviewed the key components from the previous session to put the current meeting into context.

2. Review to Sustain (for supporting memory during sessions). At key points during a meeting, such as before a consensus decision was to be made, we would review key details, including arguments from different perspectives.

3. Review to Summarize (for supporting memory between sessions). At the end of each session, we spent some time discussing and highlighting all the choices that were made 
during the meeting. We observed that this was an opportunity for new insights to be added in the context of the larger picture. This summary also served as a good way to begin the next meeting.

Though reviewing had the potential to increase familiarity with material, an amnestic's difficulty in remembering specific details was a fundamental challenge. To control for this, our meetings were planned and structured. From our interviews, we learned that amnestics often deal with problems in a structured manner to increase the chances of successfully completing a task. We thus tried to use activity structuring as much as possible. For example, in one of our meetings, content was divided into sections, and each section had one or more goals that were to be addressed. Since each section was independent of the others, there was no need to recall earlier details of the meeting at later points of the session.

\subsubsection{Creating Environmental Support}

Some research has specifically used a person's environment to reduce the demands on memory $[4,12,23]$. A simple illustration is the use of name tags that allow people to refer to one another by name. The tags remain with their wearers and constitute a part of the environment. We have utilized this theme of environmental support to architect a space for design sessions intended to support memory by reducing the demands on it during meetings.

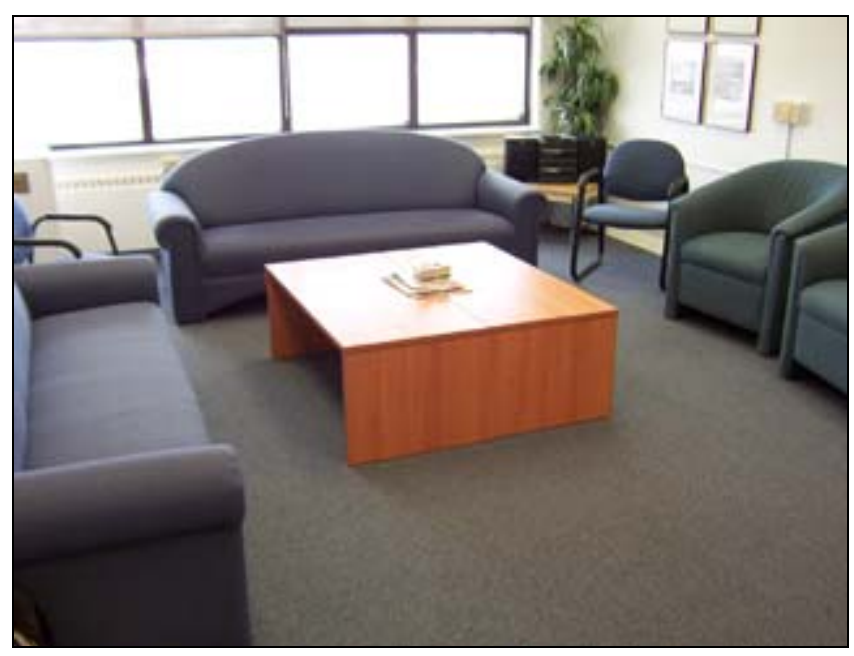

Figure 1. The Baycrest meeting room used for psychoeducational support group meetings.

The amnestic participants in our design group continue to attend weekly psychoeducational support group meetings in a meeting room (Figure 1) at Baycrest. They are familiar with the route to the building and room. We wanted to leverage this as much as possible by holding our participatory design meetings in this room instead of a completely new location. However, one problem we faced was that by holding both the psychoeducational group support sessions and design sessions in the same building, we ran the risk of making it harder for the amnestics to distinguish the temporal sequence of memories that may have been retained from the previous week. In essence, even if memories were recalled, they may be of little use if the ordering of them were mixed. When we referred to details from previous weeks, it seemed more difficult to determine from which session the idea originated. To respond to this issue, we instead arranged to use a board room at Baycrest (Figure 2) for holding our participatory design meetings.

The board room is a very distinctive space. It conveys a special feeling of importance as many people associate board rooms with prominent executive meetings. While Baycrest is a familiar location, none of the design group members have worked in the board room before.

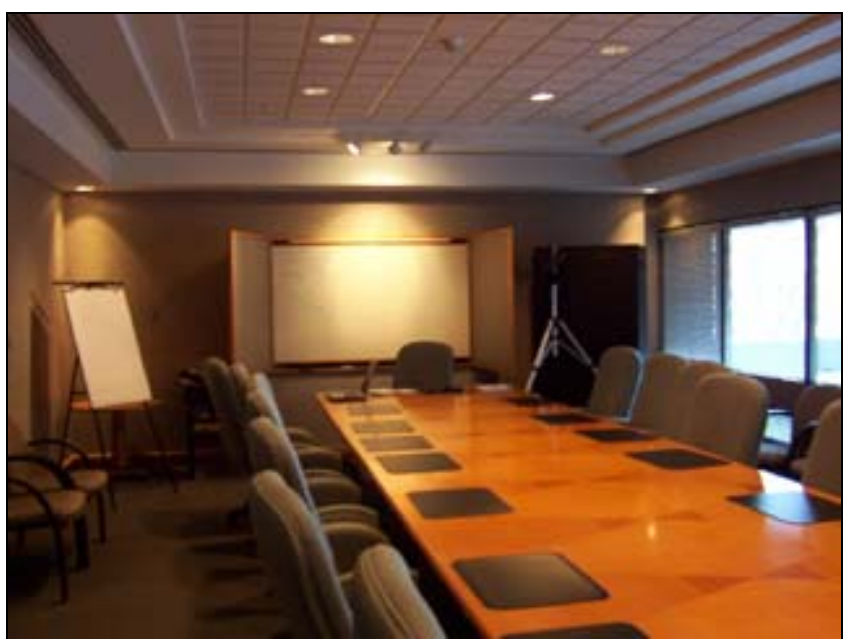

Figure 2. The Baycrest Exton board room used for design group discussions.

We argue that our choice in location for the design meetings supports memory by providing distinctive contextual cues that serve to promote discriminitability. Using a different space does introduce new issues - one point being that the unfamiliar space can lead to disorientation. To deal with this, we all met at the original familiar meeting spot and went to the board room as a group, thus removing the time consuming need to teach each individual the route to the new room. One amnestic noted early on that though the board room was an unfamiliar space, she recognized the people who were in the group from the normal weekly psychoeducational support group, and so felt grounded and comfortable, even at times when she felt a little disoriented at the meeting.

For the majority of the amnestic participants in our design meetings, details of the meeting decayed and were not retained between weeks. After several sessions, however, many recognized the location and understood that a meeting, of which they were members, took place in that space. They knew that they were selected for this work and many could, though inconsistently, recall fragments of discussion when cued with other fragments that helped to trigger the recall.

\subsubsection{Emphasizing Physical Artifacts}

As with past neuropsychological case studies [24], our field studies have shown that amnestics rely heavily on external memory aids, such as a calendar or an action item list. This is somewhat equivalent to memory triggers, for example strings on fingers, which people use to remind themselves to do something. With an amnestic, however, using a strategy such as a string on their finger will likely fail because remembering the original message attached to the trigger is difficult. Thus, though physical artifacts can aid memory, they must be used in a specific way. We 
used two different kinds of artifacts in our participatory design sessions:

1. Paper Documents. Paper documents were used extensively for guiding discussions. Some examples include: meeting agendas, summaries from past weeks, use case scenarios, and options listings. For example, whenever we had a set of choices that needed to be considered by our team, printed materials that detailed the relevant options were brought to the meeting. Oftentimes, going through each option involves a significant amount of discussion. After discussing each item in turn, an amnestic may forget some of the details presented in earlier options. Thus, having this detail readily available on paper assists the decision-making process. Though we often summarize the details by reviewing the options before making a decision, summaries tend to omit specific detail.

2. Design Artifacts. We created various physical artifacts such as storyboards where appropriate throughout our design. We often included these artifacts while summarizing our meeting by referring to salient elements of the object relevant to our discussion. We also used paper prototyping [22], which resulted in paper design artifacts (see Figure 3 for an example). These physical artifacts were brought to subsequent meetings to supplement reviews, offering a richer context to help trigger recall. From time to time, we arranged objects from past meetings in a linear fashion on the table to illustrate our progression in the design process. The long table of our board room (see Figure 2) afforded this ability.

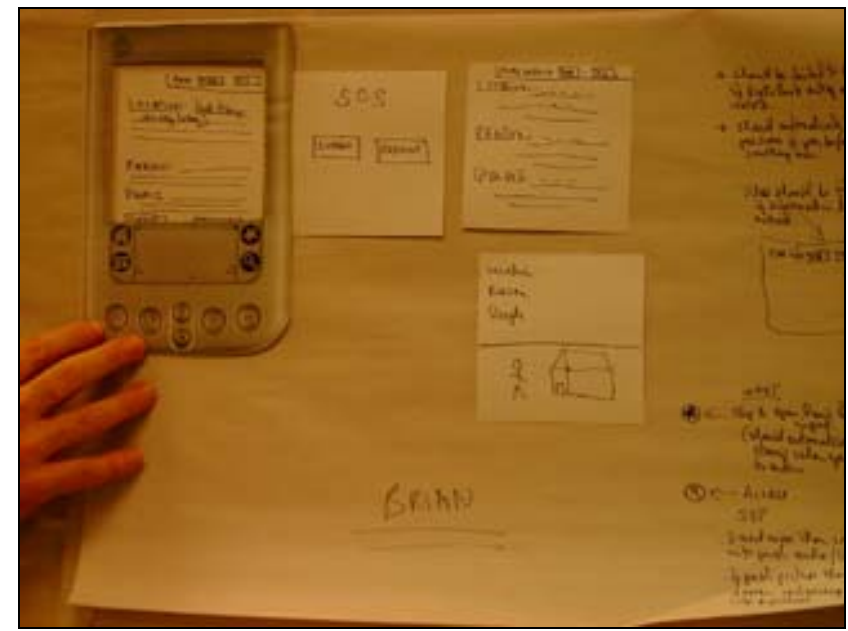

Figure 3. An example of a paper prototype design artifact.

\subsubsection{Documenting Design History}

Supporting memory between design sessions is one goal in the field of design rationale, which seeks to capture and maintain documentation detailing how designers reason and arrive at their decisions [18]. Though creating documentation can be tedious and time consuming, it becomes vital when working with amnestic individuals.

One way of supporting memory between weeks is to allow each participant to take the contents of the meeting with them when they leave, so as to allow review in between sessions. We initially wanted to pass around physical journal books, in which participants could record meeting information. However, as we have noted from our site visits as well as our own attempts at giving out homework packages, objects could be misplaced if the amnestic individuals were not trained to use them regularly. We have found that such training could take as long as 2-3 months, depending on the individual. The thought of putting the information online was considered in response to this, but this was not possible as many of the group members were not comfortable using a desktop computer, nor had convenient access to the Internet.

Before the formation of our design group, a researcher (the second author) had trained the amnestics in our team to use a Palm Pilot PDA for scheduling appointments and managing lists of action items as part of a rehabilitation program independent of our design project. This training made use of procedural memory systems that remain intact in amnestics [21] (see Figure 4). As such, each member of our design group used a Palm device on a daily basis. We wanted to utilize this training by synchronizing meeting notes into the Palm. In this way, members could take home the minutes and would have access to them throughout the week. Since the memo application was frequently used, the likelihood of reviewing the material from our meetings was reasonably good.

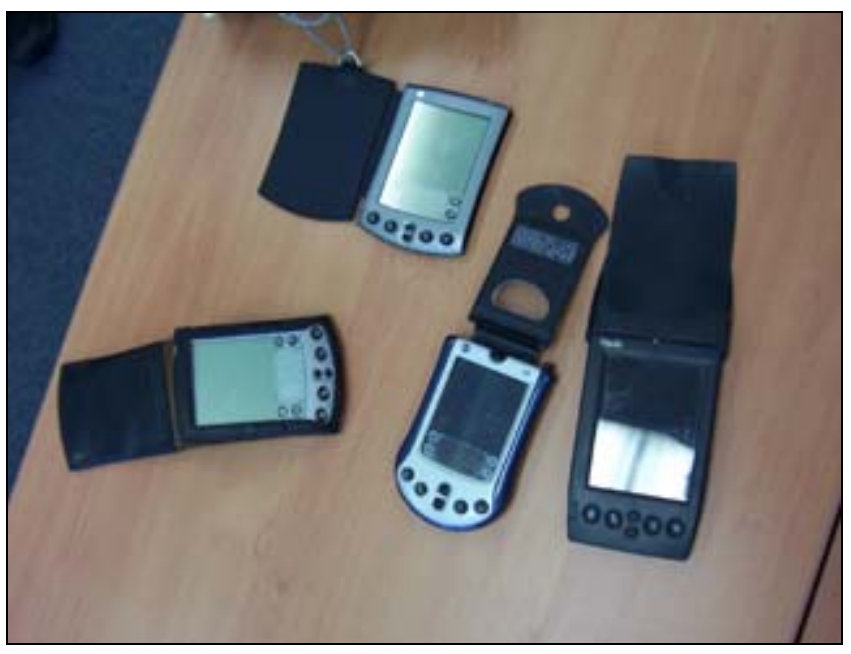

Figure 4. A few of the Personal Digital Assistants used by the amnestics on the design team.

The main problem with this idea was that typing up meeting details was something to be done at a computer after everyone left the meeting. As we held meetings every week, this meant that one week's meeting notes would not be available until the following week. We overcame this by creating two different sets of notes that were staggered in how they were distributed:

1. Summary Notes. One set of notes described the meeting summary (approximately 100-200 words) along with key homework questions, which were typed up on a laptop at the end of each meeting while the key points were being reviewed and summarized. After this was completed, the summaries were transferred from laptop to one of the Palms by HotSync. We then used the infrared beaming function of the Palms to pass the notes around to the rest of the group, thereby allowing everyone to leave the meeting with the meeting summary. Making the information immediately available was beneficial in other respects as well; one amnestic made this comment of our process: "This is great. I 
can show this to my wife. She always asks me what I did that day and I can't remember." The summary notes were kept short on purpose to avoid the necessity of reading through large amounts of text on a small screen device to get the overall understanding of what occurred. Refer to Table 3 for an example of some summary notes.

2. Detailed Meeting Minutes. The second set of notes was a more detailed version of the meeting minutes (typically 300 700 words) that included rationale and justifications for design decisions that were made. These minutes were typed up after the meeting and transferred to the Palm devices the following week. We video recorded each design meeting and occasionally added detail collected from the tapes to the minutes as necessary. Refer to Table 4 for an example of some detailed meeting minutes.

Table 3. An example of summary notes (approx 100 words).

Design Meeting 1 Summary
$===$ LOGISTICS ===
Oct 16, 2003
10:00am - $12: 15 \mathrm{pm}$
Baycrest Exton room
who: (removed)
- today's goal: project overview, discuss
expectations, discuss memory issues
=== ABOUT PROJECT ===
- peoject goal is to build/design PDA
software for amnestics
- benefits of research:
(1) develop useful device
(2) contribution to a larger impact
=== DISCUSSION OF ISSUES ENCOUNTERED ===
(1) finding way back
(2) answering phone calls
(3) use spiral notebook
(4) losing of Palm
(5) losing of Palm stylus
(6) finding information in Palm
(7) own handwriting \& memory
(8) undelete an appointment
$===$ NEXT MEETING ===
- more detailed exploration into current
practice
- role playing, give scenario to each

Table 4. An example of detailed meeting minutes (300 words).

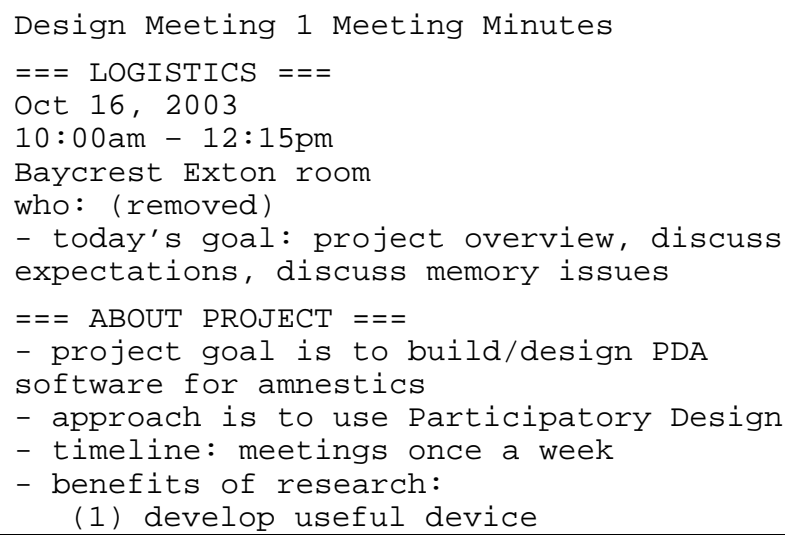

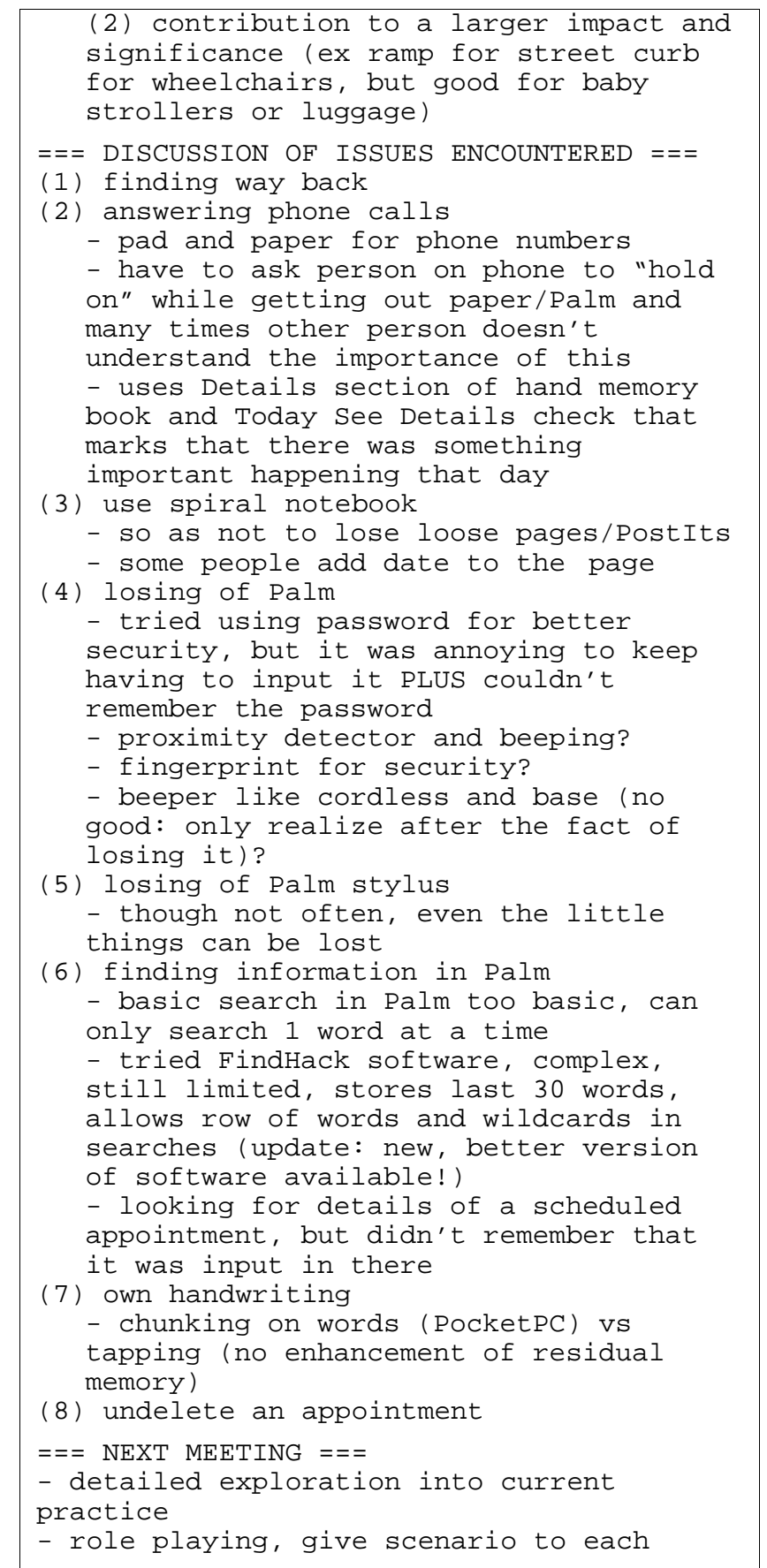

\section{DISCUSSION}

Our case study has highlighted several issues that arise when members of a participatory design team suffer from amnesia. Some of these issues are caused by discrepancies between participants' actual abilities and assumptions of cognitive functioning that are typically made in standardized design techniques. In this section we deconstruct these assumptions and present a more general discussion of designing with users who have special needs.

Among other things, cognitive impairments can affect a person's ability to remember, solve problems, communicate, process 
sensory information, maintain concentration, and plan. Cognitive deficits are variable in their effects and can make everyday tasks such as cooking, dressing, or making a phone call immensely difficult. It is important to realize that while there are other impairments unrelated to cognition that challenge assumptions of participatory design, cognitive impairments often establish fundamental barriers to design and collaboration.

We attempt to extract what we have learned from our experiences to define a framework for assessing elements of participatory design. What follows is an initial sketch of this framework followed by two specific examples from our case study demonstrating how we modified our group activities in response to two different cognitive barriers.

\subsection{When Design Partners Have Cognitive Impairments}

Traditional methods of participatory design have been primarily used with populations of normal cognitive-functioning individuals. Many of these design techniques do not explicitly take into account impairments or barriers to cognition - the result being that assumptions of capabilities are often made or taken for granted. Such assumptions must be questioned when working with individuals having cognitive impairments. The standard participatory design techniques can then become initial starting points to modify or adapt. We break this entire process down into the following four steps:

1. Assess Each Participant

2. Understand the Cognitive Deficit

3. Choose Technique and Identify Assumptions

4. Adapt, Attempt, and Refine Approach

\section{Assess Each Participant}

The first step involves an assessment of the unique abilities of each participant. It is not only important to understand how the problems affect someone on an individual basis, but also how cognitive issues may influence interactions among participants. Some cognitive disorders may co-occur and it is important to know which combinations are present in an individual.

Assessment utilizes the expertise of many disciplines including neurologists, neuropsychologist, neuropsychiatrists, rehabilitation doctors, occupational therapists, speech-language pathologists, and audiologists.

However, these assessments are often conducted in hospitals or locations in which participants do not spend much time. Interviews or ethnographic studies could be very important to better understand each participant as well as identify potential problem activities in context.

Questions that need to be answered in this step include: Are the participants aware of their own limits? Are they aware of the limits of other members in the design group? What are individual strengths and weaknesses of each person? To what degree does the impairment affect the participant? What strategies are currently being used to compensate for the cognitive impairment? What strategies are available?

\section{Understand the Cognitive Deficit}

The second step involves identifying the cognitive deficit of interest and then examining its associated qualities.

We are concerned with the questions of: What cognitive deficits are involved? Is the deficit a degradation of function or complete lack thereof? What human functions does the cognitive impairment affect? Table 5 enumerates cognitive processes which can be impaired either in isolation or in combination. Once the cognitive deficits have been identified, it is important to examine their associated attributes as relevant to the strengths and weaknesses of the participants. These attributes describe the specific details of the cognitive deficit. For example, in the case of memory, this might include: incorrect temporal sequencing of memory fragments, the degree of details remembered from events, and the duration of memory retention.

Table 5. An enumeration of the major cognitive domains and exemplars of abilities within those domains. This list is not exhaustive, but is shown to illustrate the range of cognitive abilities that can become impaired.

\begin{tabular}{|c|c|}
\hline $\begin{array}{l}\text { Cognitive } \\
\text { Domains }\end{array}$ & Cognitive Abilities \\
\hline Attention & $\begin{array}{l}\text { Focused attention } \\
\text { Divided attention } \\
\text { Withstanding distraction }\end{array}$ \\
\hline Memory & $\begin{array}{l}\text { Short-term } \\
\text { Long-term } \\
\text { Implicit } \\
\text { Explicit }\end{array}$ \\
\hline Language & $\begin{array}{l}\text { Comprehending spoken and written } \\
\text { words and sentences } \\
\text { Comprehending concepts, e.g. metaphors } \\
\text { Expressing descriptions or explanations } \\
\text { Speaking } \\
\text { Writing }\end{array}$ \\
\hline $\begin{array}{c}\text { Visuo-spatial } \\
\text { processing }\end{array}$ & $\begin{array}{l}\text { Object recognition } \\
\text { Location } \\
\text { Motion } \\
\text { Depth perception }\end{array}$ \\
\hline $\begin{array}{l}\text { Executive } \\
\text { function }\end{array}$ & $\begin{array}{l}\text { Initiation, cessation, and control of action } \\
\text { Abstract and conceptual thinking } \\
\text { Cognitive estimation and prediction } \\
\text { Cognitive flexibility } \\
\text { Response to novelty } \\
\text { Goal-directed behavior }\end{array}$ \\
\hline
\end{tabular}

\section{Choose Technique and Identify Assumptions}

This step involves deciding upon a technique and deconstructing its cognitive requirements and assumptions.

The first thing to do is choose a technique or design activity to analyze. Kensing et al. [13] and Muller et al. [19, 20] discuss how to choose the most appropriate participatory design technique to suit the task at hand. This is often the best place to start as the standard approach provides a good template and basic structure 
from which modifications can be made. Among the questions that must be answered when choosing a technique are: What are the goals of the technique? How many people are involved? What is the participation model? What phases or activities are involved in the technique?

Identifying the assumptions of a technique requires a closer inspection of its demands on the participants. In particular, what are the cognitive requirements and how are they related to the technique's goals, structure, and participation model? Some aspects of these requirements are fundamental to the activity (for example, a group discussion must involve communication), while other aspects are flexible (communication can be verbal, gestural, etc). This flexibility can suggest ways in which a technique may be modified or adapted.

\section{Adapt, Attempt, and Refine Approach}

This step involves identifying issues that may arise when attempting a technique, and then modifying it. Refinement of the approach is important to ultimately arrive at a suitable and effective solution.

Adapting the design technique is a creative process which involves using the information that has been gathered from the previous three steps and innovating new strategies to deal with the concerns. Some questions that may assist this stage are: How can human functions be supported using technology or other nontechnological practices? Can the impractical activity be avoided or the technique changed to play on the strengths of the participants? A particularly interesting question is: Are there features from other design techniques that can be useful here and if so, is it possible to integrate those features into a solution? This opens up many possibilities that include mixing a participatory design approach with a more user-centred design philosophy. To what degree can these processes be integrated and how should information between the approaches be utilized?

Attempting the technique is important to evaluate its effectiveness. However, there are consequences involved in this trail and error approach. For example, in our case study, when we evaluated our environmental support ideas, we ran into the difficulty that no amnestic was familiar with the route to the new location. This could have had very negative implications if we had tried to teach it to everyone rather than gathering the group and leading them to the new setting. It is wise to be watchful for potential consequences and conflicts before employing the technique in practice.

It is important to revise and improve the technique as necessary based on what has been learned from practice. This step of adapt, attempt, and refine is an iterative one that can be used to eventually arrive at a viable solution.

\subsection{Initiation in Case Study}

To illustrate use of this framework, we use it to analyze an actual issue that arose in our case study. Through our design sessions, we saw that a decreased initiation significantly affected our group design discussions. This initiation difficulty threatened the realization of the goal of ensuring every individual on our team had an equal voice in design.

\section{Assess Each Participant}

In our case study, two individuals in our group of eight participants had mild initiation issues, which resulted in them not bringing up their ideas during group discussion. This was a significant problem that prevented them from contributing their thoughts and voices. Both of these individuals were originally assessed by a neuropsychologist from Baycrest before the start of our design project. We found that they did not have difficulty maintaining one-on-one conversations, and had no problems ending a dialogue. We also noted that both participants were able to speak their thoughts when asked or prompted.

\section{Understand the Cognitive Deficit}

Brain injured individuals can suffer from an impaired ability to initiate, sustain, or terminate conversation. A lack of initiation is a disassociation of thought and action. It is unrelated to memory, and we had not originally anticipated that this would be a concern, but soon realized that it would affect how we conducted our design discussions.

\section{Choose Technique and Identify Assumptions}

The technique of interest is a basic activity of many participatory design activities: group discussion. When considering group discussions, one of the cornerstones of participatory design is that all participants carry an equal voice and can equally influence the design outcome by providing their opinions and thoughts. However, participants with initiation difficulties do not have an equal opportunity to take initiative in the conversation; thus there is no 'democratic dialogue' [15].

\section{Adapt, Attempt, and Refine Approach}

We wanted to equalize each participant's ability to contribute ideas. The approach we followed was borrowed from group psychotherapy. In such sessions, there is a leader who facilitates the meeting and a co-leader who watches for cues and ensures that everyone contributes. This is achieved by asking questions and making prompts at appropriate times while trying to avoid being too directive or invasive. In our group, the rehabilitation specialist (second author) carried this role as he had prior experience with this technique.

We tried out this approach in our design discussions, using the expertise of the rehabilitation specialist to read appropriate cues. We also decided that for important items during a meeting (for example, defining the main questions to address at the end of a session in preparation for the next meeting), we would impose a slightly more directive structure on the meeting in which we specifically asked each participant for their thoughts or if they might have anything to add to the discussion. The prompting was not simply directed to those having initiation issues. Instead, this technique was applied to all members of the design team so as not to single out or embarrass individuals.

We have succeeded with this approach. For example, one time after a general question to the group yielded no replies, we saw that one individual responded with thoughts and opinions when selectively asked. In this way, we encouraged participation in a controlled manner. 


\subsection{Memory in Case Study}

As we have seen in our case study, a lack of memory can cause much difficulty in the design process. The majority of our efforts have focused on minimizing the effects of such deficit and supporting memory where possible. This is the result of regarding a lack of memory as a liability in design. However, we have found a case in which a lack of memory can be considered a strength rather than a weakness. We use our framework to explore this idea further by analyzing the effects of the cognitive impairment and then deriving advantages from those effects that can be used to adapt a design technique.

\section{1-2. Assess Each Participant, Understand the Deficit}

Through our design sessions, we observed that our amnestic participants were not able to recall specific details from events happening in prior weeks. This included the very ideas that they themselves came up with. In one situation, after we presented one of our amnestic design partners with the novel concept he presented the week before, he commented, "No, I don't remember... It certainly sounds like something I would say." We found that this was a common response from others in our group.

\section{Choose Technique and Identify Assumptions}

As amnestics have difficulty recalling events and facts, when presented with a set of ideas gathered from past meetings, it can be difficult to identify who supported which ideas, or who came up with novel thoughts. This reveals an odd effect; that is, the separation of ideas from their source. The benefit of such effect is the ability to review and revisit past arguments in an unbiased way. People can criticize an idea without making the person who came out with the idea feel uncomfortable. This is a very unique property of analysis that does not commonly exist in regular participatory design meetings.

\section{Adapt, Attempt, and Refine Approach}

We supported the separation of ideas from their source by not recording names beside the ideas as the minutes and notes were typed up. It is true that fragments of details may in fact trigger recall and so an idea may help someone recollect its source, but this was not done consistently in our group.

In one incident, we observed one amnestic changing her mind between successive weeks and supporting the other side of an argument upon presentation of additional facts and thoughts. This technique would be difficult to set up with persons having normal memory because of the nature of face-to-face meetings and realtime collaborative discussions. We believe that our approach has potential to be developed and refined further.

\section{CONCLUSIONS}

We have presented a case study of participatory design with persons having amnesia, including techniques and methods that were adapted to suit the needs of our design participants. We have tried to reduce the demands made on explicit memory during the design process, and in doing so, have minimized the effects of variations in memory ability and capacity between participants. Though it was not clear how far we could push participatory design with a group of amnestics, our techniques have been successful in creating the outcomes anticipated by the process (i.e., an understanding of user needs, a specification of requirements, the development of a concept, and the high-level and low-level design of a system to be built).

This case study has led us to identify assumptions of participatory design that have been established with normal cognitivelyfunctioning populations. We have shown how some of these assumptions manifest themselves in the design process, and provide a framework that offers a practical approach toward adapting techniques.

Pushing the characteristics of design to the extreme sheds light on the participatory design process itself. Not only can this assist us when working with people who have cognitive impairments, but our case study of amnesia may also present a model for those who have normal-functioning memory. It is not difficult to imagine situations in which past meeting content is forgotten due to incomplete documentation, information overload, or simply a long break since the last meeting. Such circumstances can easily arise when participants are placed under stressful deadlines or settings. While this loss of information is not attributed to amnesia, the resulting effect is similar. We believe that examining the issues of cognitive functioning when the limits of participatory design have been stretched lead to insights into more ordinary situations.

\section{CURRENT STATUS \& FUTURE WORK}

Through our participatory design sessions, we have designed and developed a novel software tool for Personal Digital Assistants, specifically created to accommodate the needs of people having amnesia. This tool will assist amnestics when they feel lost or disoriented by providing information as to their whereabouts and their intent for being where they are.

A person having amnesia will typically follow familiar routines in their daily life, such as the same route home, because deviating from this path will often result in disorientation. Our tool enables an amnestic to grow increasingly confident and independent in exploring new locations and situations - a feat that is very difficult in current practice.

As of the submission of this paper, we are evaluating the tool that we have built. Through the design sessions, our team chose a computer platform (Palm PDA) for this tool. We have since designed the software and implemented the solution. We then evolved training techniques for interaction with the device. Researchers in the past have found that an amnestic's procedural memory system remains largely intact $[11,21]$. We have successfully utilized these preserved cognitive functions and abilities in the training of a group of amnestics. This training has given these amnestics the necessary skills to effectively use our tool. This enables us to plan more in depth evaluations of the device to better understand usage behaviours. We have already executed the first phase of our user study, and have analyzed the results. We are currently conducting the second phase of our evaluation, and intend on presenting our findings along with the system requirements, functionality, appearance, and interaction style in a future paper.

\section{ACKNOWLEDGMENTS}

We would like to thank the amnestic participants and their family members for being so open with us and for their positive attitudes and energy. We also are grateful to Michael Muller and Jaime Montemayor for initial feedback and background before the commencement of this work. Also, many thanks to Ritu Bedi, 
Krista Strickland, Jing Su, Alvin Chin, Tira Cohene, and members of the University of Toronto Dynamic Graphics Project for insightful discussions. Finally, we thank Guy Proulx, Larry Leach, and Lynda Dunal from Baycrest Centre for Geriatric Care for their ideas and support.

\section{REFERENCES}

[1] Bødker, S., Ehn, P., Kammersgaard, J., Kyng, M., \& Sundblad, Y. (1987). A UTOPIAN experience: On design of powerful computer-based tools for skilled graphic workers. In G. Bjerknes, P. Ehn, and M. Kyng (eds.), Computers and Democracy - a Scandinavian Challenge. p. 251-278.

[2] Cole, E., Dehdashti, P., Petti, L., \& Angert, M. (1994). Participatory design for sensitive interface parameters: contributions of traumatic brain injury patients to their prosthetic software. In Proceedings of the SIGCHI conference on Human factors in computing systems, p. 115116.

[3] Cole, E., \& Dehdashti, P. (1998). Computer-based cognitive prosthetics: assistive technology for the treatment of cognitive disabilities. In Proceedings of the international ACM conference on Assistive Technologies, p.11-18.

[4] Craik, F., Anderson, N., Kerr, S., \& Li, K. (1995). Memory Changes in Normal Ageing. In A. Baddeley, B. Wilson, \& F. Watts (eds.), Handbook of Memory Disorders. John Wiley \& Sons. p. 211-241.

[5] Curran, T., \& Schacter, D. (2000). Cognitive Neuropsychological Issues. In M. Farah \& T. Feinberg (eds.), Patient-Based Approaches to Cognitive Neuroscience. The MIT Press. p. 291-299.

[6] Druin, A. (1999). Cooperative inquiry: Developing new technologies for children with children. In Proceedings of the SIGCHI conference on Human factors in computing systems, ACM Press. p. 223-230.

[7] Druin, A. (1999). The Role of Children in the Design of New Technology, Behaviour and Information Technology (BIT), 21 (1), p. 1-25.

[8] Druin, A., Bederson, B., Boltman, A., Miura, A., KnottsCallahan, D., and Platt, M. (1998). Children as Our Technology Design Partners. In A. Druin (ed.), The Design of Children's Technology: How we design and why?, Morgan Kaufmann, p. 51-72.

[9] Farber, A., Druin, A., Chipman, G., Julian, D., \& Somashekhar, S. (2002). How Young Can Our Design Partners Be? In Proceedings of the Participatory Design Conference, p. 272-277.

[10] Fischer, G., \& Sullivan, J. Jr. (2002). Human-Centered Public Transportation Systems for Persons with Cognitive Disabilities. In Proceedings of the Participatory Design Conference, p. 194-198.

[11] Graf, P., \& Schacter, D. L. (1985). Implicit and explicit memory for new associations in normal and amnesic subjects. Journal of Experimental Psychology: Learning, Memory and Cognition, 11, p. 501-518.
[12] Kapur, N. (1995). Memory Aids in the Rehabilitation of Memory Disordered Patients. In A. Baddeley, B. Wilson, \& F. Watts (eds.), Handbook of Memory Disorders. John Wiley \& Sons. p. 533-556.

[13] Kensing, J., Simonsen, J., Bødker, S. (1996). MUST: a Method for Participatory Design. In Proceedings of the Participatory Design Conference, p. 129-140.

[14] LoPresti, E.F. Mihailidis, A., Kirsch, N. (2004). Assistive technology for cognitive rehabilitation: State of the art. Neuropsychological Rehabilitation, 14 (1/2), p. 5-39.

[15] Madsen, K. (1996). Initiative in Participatory Design. In Proceedings of the Participatory Design Conference, p. 223230.

[16] McGrenere, J., Davies, R., Findlater, L., Graf, P., Klawe, M., Moffatt, K., Purves, B., \& Yang, S. (2003). Insights from the Aphasia Project: Designing Technology for and with People Who Have Aphasia. In Proceedings of the ACM Conference on Universal Usability, p. 112-118.

[17] Moffatt, K., McGrenere, J., Purves, B., Klawe, M. (2004). The participatory design of a sound and image enhanced daily planner for people with aphasia. In the Proceedings of the SIGCHI conference on Human factors in computing system, p. 407-414.

[18] Moran, P., \& Carroll J. (1996). Design Rationale: Concepts, Techniques, and Use. Lawrence Erlbaum Associates.

[19] Muller, M., Halkswanter, J. \& Dayton, T. (1997). Participatory practices in the software lifecycle. In M. Helander, T. Landauer \& P. Prabhu (eds.), Handbook of Human-Computer Interaction. $2^{\text {nd }}$ edition. Elsevier. p. $255-$ 313.

[20] Muller, M. (2002). Participatory Design: The Third Space. In J. Jacko \& A. Sears (eds.), The Human-Computer Interaction Handbook: Fundamentals, Evolving Technologies and Emerging Applications. Lawrence Erlbaum Associates.

[21] Squire, L.R. (1982). The neuropsychology of human memory. Annual Review of Neuroscience, 5, p. 241-273.

[22] Snyder, C. (2003). Paper Prototyping: The Fast and Easy Way to Design and Refine User Interfaces, Morgan Kaufmann Publishers.

[23] Sohlberg, M. M. \& Mateer, C. A. (2001). Cognitive Rehabilitation: An integrative neuropsychological approach. The Guilford Press.

[24] Wilson, B. (1999). Case Studies in Neuropsychological Rehabilitation, Oxford University Press.

[25] Wilson, B. (2002). Memory Rehabilitation. In L. Squire, \& D. Schacter (eds.), Neuropsychology of Memory. $3^{\text {rd }}$ edition. The Guilford Press. p. 263-272.

[26] Wu, M. (2004). The Participatory Design of an Orientation Aid for People with Amnesia. M.Sc. Thesis. Department of Computer Science, University of Toronto. 